J. Indones. Math. Soc.

Vol. 17, No. 1 (2011), pp. 17-28.

\title{
SYSTEMS OF FUZZY NUMBER MAX-PLUS LINEAR EQUATIONS
}

\author{
M. Andy Rudhito ${ }^{1,5}$, Sri Wahyuni ${ }^{2}$, Ari Suparwanto ${ }^{3}$ and Frans \\ SusILO $^{4}$ \\ ${ }^{1}$ Department of Mathematics Gadjah Mada University Yogyakarta \\ Indonesia, arudhito@yahoo.co.id \\ ${ }^{2}$ Department of Mathematics Gadjah Mada University Yogyakarta \\ Indonesia, swahyuni@ugm.ac.id \\ ${ }^{3}$ Department of Mathematics Gadjah Mada University Yogyakarta \\ Indonesia, ari_suparwanto@yahoo.com \\ ${ }^{4}$ Department of Mathematics Sanata Dharma University Yogyakarta \\ Indonesia, fsusilo@staff.usd.ac.id
}

\begin{abstract}
This paper discusses the solution of systems of fuzzy number max-plus linear equations through the greatest fuzzy number subsolution of the system. We show that if entries of each column of the coefficient matrix are not equal to infinite, the system has the greatest fuzzy number subsolution. The greatest fuzzy number subsolution of the system could be determined by first finding the greatest interval subsolution of the alpha-cuts of the system and then modifying it if needed, such that each its components is a family of alpha-cut of a fuzzy number. Then, based on the Decomposition Theorem on Fuzzy Set, we can determine the membership function of the elements of greatest subsolution of the system. If the greatest subsolution satisfies the system then it is a solution of the system.

Key words: Max-Plus algebra, system of linear equations, fuzzy number.
\end{abstract}

\footnotetext{
${ }^{5}$ Permanent Address: Department of Mathematics and Natural Sciences Education Sanata Dharma University Yogyakarta Indonesia

2000 Mathematics Subject Classification: 08A72, 15A80, 15B15, 34A30.

Received: 14-10-2010, revised: 10-03-2011, accepted: 12-03-2011.
} 


\begin{abstract}
Abstrak. Artikel ini membahas tentang penyelesaian sistem persamaan linear max-plus bilangan kabur melalui subpenyelesaian terbesar bilangan kabur sistem tersebut. Dapat ditunjukkan bahwa untuk matriks persegi, dengan komponen pada setiap kolom tidak semuanya sama dengan tak hingga, mempunyai subpenyelesaian terbesar bilangan kabur. Subpenyelesaian terbesar bilangan kabur sistem dapat ditentukan dengan terlebih dahulu menentukan vektor subpenyelesaian terbesar interval potongan-alpha sistem tersebut dan jika diperlukan dilakukan modifikasi sedemikian hingga setiap komponennya merupakan potongan-alpha suatu bilangan kabur. Selanjutnya, dengan berdasarkan Teorema Dekomposisi pada Himpunan Kabur, dapat ditentukan fungsi keanggotaan komponen subpenyelesaian terbesar sistem. Jika subpenyelesaian terbesar memenuhi sistem, maka subpenyelesaian terbesar tersebut merupakan penyelesaian sistem.
\end{abstract}

Kata kunci: Aljabar max-plus, sistem persamaan linear, bilangan kabur

\title{
1. Introduction
}

The max-plus algebra can be used to model and analyze networks, like the project scheduling, production system, queuing networks, etc [1], [3], [4]. The networks modeling with max-plus algebra approach is usually a system of maxplus linear equations and it can be written as a matrix equation $A \otimes \boldsymbol{x}=\boldsymbol{b}$ where $\boldsymbol{x}$ and $\boldsymbol{b}$ are input vector and output vector respectively.

Recently, the fuzzy networks modeling have been developed. In this paper, the fuzzy networks refer to networks whose activity times are fuzzy numbers. The fuzzy scheduling are as in [2], [10] and the fuzzy queueing networks as in [7]. When we follow the notions of modeling and analyzing fuzzy networks with max-plus algebra approach, for the input-output fuzzy system we will use systems of fuzzy number max-plus linear equations. For this reason, this paper will discuss existence and computation of solutions of system $\tilde{A} \tilde{\otimes} \tilde{\boldsymbol{x}}=\tilde{\boldsymbol{b}}$ of fuzzy number max-plus linear equations.

The solution of the fuzzy relational equation has been investigated by many researchers. Among their results, are the minimal solution of a fuzzy relational equation with the supinf composite operation [8], the complete set of minimal solutions for fuzzy relational equations with max-product composition [11], the solutions for fuzzy relational equations with supmin composition [12], and the solutions for fuzzy relational equations with supinf composition [9]. The solutions for fuzzy relational equations with max-plus composition will be discussed in this paper.

We first review some basic concepts of max-plus algebra, matrices over maxplus algebra, and the solution of system of max-plus linear equations $A \otimes \boldsymbol{x}=\boldsymbol{b}$. Further details can be found in [1].

Let $\mathbf{R}_{\varepsilon}:=\mathbf{R} \cup\{\varepsilon\}$ be the set of all real numbers and $\varepsilon:=-\infty$. Defined two operations on $\mathbf{R}_{\varepsilon}$ such that for every $a, b \in \mathbf{R}$,

$$
a \oplus b:=\max (a, b), a \otimes b:=a+b .
$$


Then $(\mathbf{R}, \oplus, \otimes)$ is a commutative idempotent semiring whose neutral element $\varepsilon=$ $-\infty$ and unity element $e=0$. Moreover, $(\mathbf{R}, \oplus, \otimes)$ is a semifield, that is $(\mathbf{R}, \oplus, \otimes)$ is a commutative semiring, where for every $a \in \mathbf{R}$ there exist $-a$ such that $a \otimes(-a)=$ 0 . Then, $(\mathbf{R}, \oplus, \otimes)$ is called the max-plus algebra, and is written as $\mathbf{R}_{\max }$. The algebra $\mathbf{R}_{\max }$ has no zero divisors, that is for every $x, y \in \mathbf{R}$, if $x \otimes y=\varepsilon$, then $x=\varepsilon$ or $y=\varepsilon$. The relation " $\preceq_{m}$ " on $\mathbf{R}_{\max }$ defined by $x \preceq_{m} y$ if $x \oplus y=y$, is a partial order on $\mathbf{R}_{\max }$. The operations on $\mathbf{R}_{\max }$ are consistent with respect to the order $\preceq_{m}$, that is for every $a, b, c \in \mathbf{R}_{\text {max }}$, if $a \preceq_{m} b$, then $a \oplus c \preceq_{m} b \oplus c$ and $a \otimes c \preceq_{m} b \otimes c$. We define $x^{0}:=0, x^{k}:=x \otimes x^{k-1}$ and $\varepsilon^{k}:=\varepsilon$, for $k=1,2, \ldots$.

The operations $\oplus$ and $\otimes$ on $\mathbf{R}_{\max }$ can be extended to the set in $\mathbf{R}_{\max }^{m \times n}$, where $\mathbf{R}_{\max }^{m \times n}:=\left\{A=\left(A_{i j}\right) \mid A_{i j} \in \mathbf{R}_{\max }\right.$, for $i=1,2, \ldots, m$ and $\left.j=1,2, \ldots, n\right\}$. Specifically, for $A, B \in \mathbf{R}_{\max }^{n \times n}$ we define

$$
(A \oplus B)_{i j}=A_{i j} \oplus B_{i j},(A \otimes B)_{i j}=\bigoplus_{k=1}^{n} A_{i k} \otimes B_{k j} .
$$

We also define matrix $\Upsilon \in \mathbf{R}_{\max }^{m \times n}$, with $(\Upsilon)_{i j}:=\varepsilon$ for every $i$ and $j$, and matrix $E \in \mathbf{R}_{\max }^{n \times n}$, with $(E)_{i j}:=\left\{\begin{array}{l}0 \text { if } i=j \\ \varepsilon \text { if } i \neq j\end{array}\right.$. We can show that $\left(\mathbf{R}_{\max }^{m \times n}, \oplus\right)$ is an idempotent commutative semigroup, $\left(\mathbf{R}_{\max }^{n \times n}, \oplus, \otimes\right)$ is an idempotent semiring whose neutral element is the matrix $\Upsilon$ and unity element is the matrix $E$, and $\mathbf{R}_{\max }^{m \times n}$ is a semimodule over $\mathbf{R}_{\max }$.

For any matrix $A \in \mathbf{R}_{\max }^{n \times n}$, define $A^{0}=E_{n}$ and $A^{k}:=A \otimes A^{k-1}$ for $k=$ $1,2, \ldots$. The relation " $\preceq_{m}$ " which is define in $\mathbf{R}_{\max }^{m \times n}$ by $A \preceq B$ if $A \oplus B=B$, is a partial order on $\mathbf{R}_{\max }^{m \times n}$. In $\left(\mathbf{R}_{\max }^{n \times n}, \oplus, \otimes\right)$, operations $\oplus$ and $\otimes$ are consistent with respect to the order $\preceq_{m}$, that is for every $A, B, C \in \mathbf{R}_{\max }^{m \times n}$ if $A \preceq B$, then $A \oplus C \preceq B \oplus C$ and $A \otimes C \preceq B \otimes C$.

Define $\mathbf{R}_{\max }^{n}:=\left\{\left[x_{1}, x_{2}, \ldots, x_{n}\right]^{T} \mid x_{i} \in \mathbf{R}_{\max }, i=1,2, \ldots, n\right\}$. Note that $\mathbf{R}_{\max }^{n}$ can be viewed as $\mathbf{R}_{\max }^{n \times 1}$. The elements of $\mathbf{R}_{\max }^{n}$ are called vectors over $\mathbf{R}_{\max }$ or shortly vectors.

Definition 1.1. Given $A \in \mathbf{R}_{\max }^{m \times n}$ and $\boldsymbol{b} \in \mathbf{R}_{\max }^{m}$, a vector $\dot{\boldsymbol{x}}$ is called subsolution of the system of max-plus linear equations $A \otimes \boldsymbol{x}=\boldsymbol{b}$ if $A \otimes \boldsymbol{x} \preceq \boldsymbol{b}$.

Definition 1.2. A subsolution $\hat{\boldsymbol{x}}$ of the system $A \otimes \boldsymbol{x}=\boldsymbol{b}$ is called the greatest subsolutions of the system $A \otimes \boldsymbol{x}=\boldsymbol{b}$ if $\boldsymbol{x} \preceq \hat{\boldsymbol{x}}$ for every subsolution $\boldsymbol{x}$ of the system $A \otimes \boldsymbol{x}=\boldsymbol{b}$.

Theorem 1.3. [1] Given $A \in \mathbf{R}_{\max }^{m \times n}$ with the entries of each column are not all equal to $\varepsilon$ and $\boldsymbol{b} \in \mathbf{R}_{\max }^{m}$, then the greatest subsolution of system $A \otimes \boldsymbol{x}=\boldsymbol{b}$ exists and is given by $\hat{\boldsymbol{x}}=-\left(A^{T} \otimes(-\boldsymbol{b})\right)$. 


\section{Main Results}

We begin the discussion by developing some basic concepts of interval maxplus algebra, matrices over interval max-plus algebra, and solutions of systems of interval max-plus linear equations. The concepts are developed based on [6].

A (closed) interval $\mathrm{x}$ in $\mathbf{R}_{\max }$ is a subset of $\mathbf{R}_{\max }$ of the form

$$
[x]=[\underline{x}, \bar{x}]=\left\{x \in \mathbf{R}_{\max } \mid \underline{x} \preceq_{m} x \preceq_{m} \bar{x}\right\} .
$$

The interval $[x] \in \mathbf{R}_{\max }$ is called max-plus interval or shortly interval. For intervals $[x]$ and $[y],[x] \subseteq[y]$ if and only if $\underline{y} \preceq_{m} \underline{x} \preceq_{m} \bar{x} \preceq_{m} \bar{y}$. The number $x \in \mathbf{R}_{\max }$ can be represented as interval $[x, x]$. Define $I(\mathbf{R})_{\varepsilon}:=\left\{[x]=[\underline{x}, \bar{x}] \mid \underline{x}, \bar{x} \in \mathbf{R}, \varepsilon \preceq_{m} \underline{x} \preceq_{m}\right.$ $\bar{x}\} \cup\{[\varepsilon]\}$, where $[\varepsilon]:=[\varepsilon, \varepsilon]$. Define two operations $\bar{\oplus}$ and $\bar{\otimes}$ by

$$
[x] \bar{\oplus}[y]:=[\underline{x} \oplus \underline{y}, \bar{x} \oplus \bar{y}],[x] \bar{\otimes}[y]:=[\underline{x} \otimes \underline{y}, \bar{x} \otimes \bar{y}]
$$

for every $[x],[y] \in I(\mathbf{R})_{\varepsilon}$. We can show that $I(\mathbf{R})_{\varepsilon}$ is closed with respect to the operations $\bar{\oplus}$ and $\bar{\otimes}$. Moreover, $\left(I(\mathbf{R})_{\varepsilon}, \bar{\oplus}, \bar{\otimes}\right)$ is a comutative idempotent semiring with neutral element $[\varepsilon]$ and unity element $[0]=[0,0]$. This comutative idempotent semiring $\left(I(\mathbf{R})_{\varepsilon}, \bar{\oplus}, \bar{\otimes}\right)$ is called the interval max-plus algebra and is written as $I(\mathbf{R})_{\max }$. Relation " $\preceq_{I m}$ " defined on $I(\mathbf{R})_{\max }$ as $[x] \preceq_{\text {Im }}[y]$ if $[x] \bar{\oplus}[y]=[y]$, is a partial order on $I(\mathbf{R})_{\max }$. Notice that $[x] \bar{\oplus}[y]=[y]$ if and only if $\underline{x} \preceq_{m} \underline{y}$ and $\bar{x} \preceq_{m} \bar{y}$.

Define $I(\mathbf{R})_{\max }^{m \times n}:=\left\{[A]=\left([A]_{i j}\right) \mid[A]_{i j} \in I(\mathbf{R})_{\max }\right.$, for $i=1,2, \ldots, m$ and $j=1,2, \ldots, n\}$. The elements of $I(\mathbf{R})_{\max }^{m \times n}$ are called matrix over interval maxplus algebra or shortly interval matrices. Matrices $[A],[B] \in I(\mathbf{R})_{\max }^{m \times n}$ are equal if $[A]_{i j}=[B]_{i j}$, that is if $[A]_{i j}=[B]_{i j}$ and $\overline{[A]_{i j}}=\overline{[B]_{i j}}$ for every $i$ and $j$. The

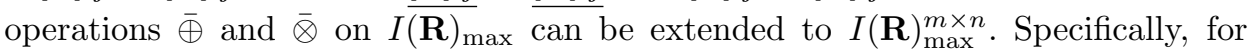
$[A],[B] \in I(\mathbf{R})_{\max }^{n \times n}$ we define

$$
([A] \bar{\oplus}[B])_{i j}=[A]_{i j} \bar{\oplus}[B]_{i j},([A] \bar{\otimes}[B])_{i j}=\bigoplus_{k=1}^{n}[A]_{i k} \bar{\otimes}[B]_{k j} .
$$

Then $\left(I(\mathbf{R})_{\max }^{m \times n}, \bar{\oplus}, \bar{\otimes}\right)$ is an idempotent semiring whose neutral element is the matrix $[\Upsilon]$, with $([\Upsilon])_{i j}:=[\varepsilon]$ for every $i$ and $j$, and unity element is the matrix $[E]$, with $([E])_{i j}:=\left\{\begin{array}{c}{[0] \text { if } i=j} \\ {[\varepsilon] \text { if } i \neq j}\end{array}\right.$. We can also show that $I(\mathbf{R})_{\max }^{m \times n}$ is a semimodule over $I(\mathbf{R})_{\max }$.

For any matrix $[A] \in I(\mathbf{R})_{\max }^{m \times n}$, define the matrices $\underline{A}=\left([A]_{i j}\right) \in \mathbf{R}_{\max }^{m \times n}$ and $\bar{A}=\left(\overline{[A]_{i j}}\right) \in \mathbf{R}_{\max }^{m \times n}$, which are called lower bound matrices and upper bound matrices of $[\mathrm{A}]$, respectively. Define matrix interval of $[A]$, as $[\underline{A}, \bar{A}]=$ $\left\{A \in \mathbf{R}_{\max }^{m \times n} \mid \underline{A} \preceq_{m} A \preceq_{m} \bar{A}\right\}$ and $I\left(\mathbf{R}_{\max }^{m \times n}\right)_{b}=\left\{[\underline{A}, \bar{A}] \mid[A] \in I(\mathbf{R})_{\max }^{m \times n}\right\}$. Also for $[\underline{A}, \bar{A}],[\underline{B}, \bar{B}] \in I\left(\mathbf{R}_{\max }^{n \times n}\right)_{b}$ we define

$$
[\underline{A}, \bar{A}] \bar{\oplus}[\underline{B}, \bar{B}]=[\underline{A} \oplus \underline{B}, \bar{A} \oplus \bar{B}],[\underline{A}, \bar{A}] \bar{\otimes}[\underline{B}, \bar{B}]=[\underline{A} \otimes \underline{B}, \bar{A} \otimes \bar{B}] .
$$

The matrices interval $[\underline{A}, \bar{A}],[\underline{B}, \bar{B}] \in I\left(\mathbf{R}_{\max }^{n \times n}\right)_{b}$ are equal if $\underline{A}=\underline{B}$ and $\bar{A}=$ $\bar{B}$. We can show that $\left(I\left(\mathbf{R}_{\max }^{n \times n}\right)_{b}, \bar{\oplus}, \bar{\otimes}\right)$ is an idempotent semiring with neutral 
element $[\Upsilon, \Upsilon]$ and the unity element $[E, E]$. We can also show that $I\left(\mathbf{R}_{\max }^{n \times n}\right)_{b}$ is a semimodule over $I(\mathbf{R})_{\max }$.

The semiring $\left(I(\mathbf{R})_{\max }^{n \times n}, \bar{\oplus}, \bar{\otimes}\right)$ is isomorphic with semiring $\left(I\left(\mathbf{R}_{\max }^{n \times n}\right)_{b}, \bar{\oplus}, \bar{\otimes}\right)$. Define a mapping $f$, where $f([A])=[\underline{A}, \bar{A}]$ for every $[A] \in I(\mathbf{R})_{\max }^{n \times n}$. Also, the semimodule $I(\mathbf{R})_{\text {max }}^{n \times n}$ is isomorphic with semimodule $I\left(\mathbf{R}_{\max }^{n \times n}\right)_{b}$. Hence, for every matrices interval $[A] \in I(\mathbf{R})_{\max }^{n \times n}$ we can determine matrices interval $[\underline{A}, \bar{A}] \in I\left(\mathbf{R}_{\max }^{n \times n}\right)_{b}$. Conversely, for every $[\underline{A}, \bar{A}] \in I\left(\mathbf{R}_{\max }^{n \times n}\right)_{b}, \underline{A}, \bar{A} \in \mathbf{R}_{\max }^{n \times n}$, such that $\left[\left[\underline{A}_{i j}, \overline{[A}_{i j}\right] \in\right.$ $I(\mathbf{R})_{\max }$ for every $i$ and $j$. The matrix interval $[\underline{A}, \bar{A}]$ is called matrix interval associated with the interval matrix $[\mathrm{A}]$ and is written as $[A] \approx[\underline{A}, \bar{A}]$. So we have

$$
[A] \bar{\oplus}[B] \approx[\underline{A} \oplus \underline{B}, \bar{A} \oplus \bar{B}],[A] \bar{\otimes}[B] \approx[\underline{A} \otimes \underline{B}, \bar{A} \otimes \bar{B}] .
$$

Define $I(\mathbf{R})_{\max }^{m \times n}:=\left\{[\mathbf{x}]=\left[[x]_{1},[x]_{2}, \ldots,[x]_{n}\right]^{T} \mid[x]_{i} \in I(\mathbf{R})_{\max }, i=1,2, \ldots, n\right\}$. The Elements of $I(\mathbf{R})_{\max }^{m \times n}$ are called interval vectors over $I(\mathbf{R})_{\max }$ or shortly interval vectors.

Definition 2.1. Given $[A] \in I(\mathbf{R})_{\max }^{m \times n}$ and $[\boldsymbol{b}] \in I(\mathbf{R})_{\max }^{m}$. An interval vector $[\boldsymbol{x}]^{*} \in I(\mathbf{R})_{\max }^{n}$ is called an interval solution of interval system $[A] \bar{\otimes}[\boldsymbol{x}]=[\boldsymbol{b}]$ if $[\boldsymbol{x}]^{*}$ satisfies $[A] \bar{\otimes}[\boldsymbol{x}]^{*}=[\boldsymbol{b}]$. An interval vector $[\boldsymbol{x}] \in I(\mathbf{R})_{\max }^{n}$ is called interval subsolution of the system if $[A] \bar{\otimes}[\dot{\boldsymbol{x}}] \preceq_{\operatorname{Im}}[\boldsymbol{b}]$.

Definition 2.2. Given $[A] \in I(\mathbf{R})_{\max }^{m \times n}$ and $[\boldsymbol{b}] \in I(\mathbf{R})_{\max }^{m}$. An interval vector $[\hat{\boldsymbol{x}}] \in I(\mathbf{R})_{\max }^{n}$ is called the greatest interval subsolution of interval system $[A] \bar{\otimes}[\boldsymbol{x}]=[\boldsymbol{b}]$ if $[\hat{\boldsymbol{x}}] \preceq_{\text {Im }}[\hat{\boldsymbol{x}}]$ for every interval subsolution $[\hat{\boldsymbol{x}}]$ of $[A] \bar{\otimes}[\boldsymbol{x}]=[\boldsymbol{b}]$.

Theorem 2.3. If $[A] \in I(\mathbf{R})_{\max }^{m \times n}$ with the entries of each column are not all equal to $[\varepsilon]$ and $[\boldsymbol{b}] \in I(\mathbf{R})_{\max }^{m}$, with $[A] \approx[\underline{A}, \bar{A}]$ and $[\boldsymbol{b}] \approx[\underline{\boldsymbol{b}}, \overline{\boldsymbol{b}}]$, then the interval vector $[\hat{\boldsymbol{x}}] \approx[\underline{\hat{\boldsymbol{x}}}, \overline{\hat{\boldsymbol{x}}}]$, with $\underline{\hat{\boldsymbol{x}}}_{i}=\min \left\{-\left(\underline{A}^{T} \otimes(-\underline{\boldsymbol{b}})\right)_{i},-\left(\bar{A}^{T} \otimes(-\overline{\boldsymbol{b}})\right)_{i}\right\}$ and $\overline{\hat{\boldsymbol{x}}}=-\left(\bar{A}^{T} \otimes(-\overline{\boldsymbol{b}})\right)$ is the greatest interval subsolution of the interval system $[A] \bar{\otimes}[\boldsymbol{x}]=[\boldsymbol{b}]$.

Proof. Since the entries of each column of matrix $[\mathrm{A}]$ are not all equal to $[\varepsilon]$ for every $A \in[\underline{A}, \bar{A}]$, the entries of each column of matrix $A$ are not all equal to $\varepsilon$. According to Theorem 1.3 for every $A \in[\underline{A}, \bar{A}]$ and for every $\boldsymbol{b} \in[\underline{\boldsymbol{b}}, \overline{\boldsymbol{b}}]$, the vector $\hat{\boldsymbol{x}}=-\left(A^{T} \otimes(-\boldsymbol{b})\right)$ is the greatest subsolution of $A \otimes \boldsymbol{x}=\boldsymbol{b}$. Especially for the system $\underline{A} \otimes \underline{\boldsymbol{x}}=\underline{\boldsymbol{b}}$, the greatest subsolution is $\underline{\hat{\boldsymbol{x}}}=-\left(\underline{A}^{T} \otimes(-\underline{\boldsymbol{b}})\right)$, and for the system $\bar{A} \otimes \bar{x}=\bar{b}$ is $\hat{\boldsymbol{x}}=-\left(\bar{A}^{T} \otimes(-\overline{\boldsymbol{b}})\right)$. Let $[\hat{\boldsymbol{x}}] \approx[\underline{\hat{\boldsymbol{x}}}, \overline{\hat{\boldsymbol{x}}}]$, with $\underline{\hat{\boldsymbol{x}}}_{i}=$ $\min \left\{-\left(\underline{A}^{T} \oplus(-\underline{\boldsymbol{b}})\right)_{i},-\left(\bar{A}^{T} \oplus(-\overline{\boldsymbol{b}})\right)_{i}\right\}$ and $\overline{\hat{\boldsymbol{x}}}=-\left(\bar{A}^{T} \oplus(-\overline{\boldsymbol{b}})\right)$.

(i) If $-\left(\underline{A}^{T} \otimes(-\underline{\boldsymbol{b}})\right) \preceq_{m}-\left(\bar{A}^{T} \otimes(-\underline{\boldsymbol{b}})\right)$ then $\underline{\hat{\boldsymbol{x}}}=-\left(\underline{A}^{T} \otimes(-\underline{\boldsymbol{b}})\right)$. Since $\underline{\hat{\boldsymbol{x}}}$ and $\overline{\boldsymbol{x}}$ are the subsolution of $\underline{A} \otimes \underline{\boldsymbol{x}}=\underline{\boldsymbol{b}}$ and $\bar{A} \otimes \overline{\boldsymbol{x}}=\overline{\boldsymbol{b}}$ respectively, $\underline{A} \otimes \underline{\boldsymbol{x}} \preceq_{m} \underline{\boldsymbol{b}}$ and $\bar{A} \otimes \overline{\boldsymbol{x}} \preceq_{m} \overline{\boldsymbol{b}}$ hold. It is followed that $[\underline{A} \otimes \underline{\boldsymbol{x}}, \bar{A} \otimes \overline{\boldsymbol{x}}] \preceq_{I m}[\underline{\boldsymbol{b}}, \overline{\boldsymbol{b}}]$, or $[\hat{A}] \bar{\otimes}[\hat{\boldsymbol{x}}] \preceq_{I m}[\boldsymbol{b}]$. Thus $[\hat{\boldsymbol{x}}]$ is a subsolution of $[A] \bar{\otimes}[\boldsymbol{x}]=[\boldsymbol{b}]$. Let the interval vector $[\hat{\boldsymbol{x}}] \in I(\mathbf{R})_{\max }^{n}$ be an interval subsolution of $[A] \bar{\otimes}[\boldsymbol{x}]=[\boldsymbol{b}]$, then $[A] \bar{\otimes}[\dot{\boldsymbol{x}}] \preceq_{I m}[\boldsymbol{b}]$ or $[\underline{A}, \bar{A}] \bar{\otimes}[\underline{\underline{\mathbf{x}}}, \overline{\mathbf{x}}] \preceq_{I m}$ $[\boldsymbol{b}]$ or $[\underline{A} \otimes \underline{x}, \bar{A} \otimes \bar{x}] \preceq_{I m}[\underline{\boldsymbol{b}}, \overline{\boldsymbol{b}}]$, that means $\underline{A} \otimes \underline{x} \preceq_{m} \underline{\boldsymbol{b}}$ and $\bar{A} \otimes \bar{x} \preceq_{m} \overline{\boldsymbol{b}}$. Hence 
$\underline{\underline{x}}$ and $\bar{x}$ is a subsolution of $\underline{A} \otimes \underline{\boldsymbol{x}}=\underline{\boldsymbol{b}}$ and $\bar{A} \otimes \bar{x}=\bar{b}$, respectively. Since $\underline{\hat{\boldsymbol{x}}}$ and $\overline{\overline{\hat{\boldsymbol{x}}}}$ are the greatest subsolution of $\underline{A} \otimes \underline{\boldsymbol{x}}=\underline{\boldsymbol{b}}$ and $\bar{A} \otimes \overline{\boldsymbol{x}}=\overline{\boldsymbol{b}}$, respectively, $\underline{\boldsymbol{x}} \preceq_{m} \underline{\hat{\boldsymbol{x}}}$ and $\overline{\boldsymbol{x}} \preceq_{m} \overline{\hat{\boldsymbol{x}}}$. It follows that $[\underline{\dot{x}}, \overline{\overline{\boldsymbol{x}}}] \preceq_{I m}[\underline{\hat{\boldsymbol{x}}}, \overline{\hat{\boldsymbol{x}}}]$. Thus, we can prove that $[\hat{\boldsymbol{x}}] \approx$ $\left[-\left(\underline{A}^{T} \otimes(-\underline{\boldsymbol{b}})\right),-\left(\bar{A}^{T} \otimes(-\overline{\boldsymbol{b}})\right)\right]$ is the greatest subsolution of $[A] \bar{\otimes}[\boldsymbol{x}]=[\boldsymbol{b}]$.

(ii) If $-\left(\underline{A}^{T} \otimes(-\underline{\boldsymbol{b}})\right) \varliminf_{m}-\left(\bar{A}^{T} \otimes(-\underline{\boldsymbol{b}})\right)$, then for $[\hat{\boldsymbol{x}}] \approx\left[\underline{\hat{\boldsymbol{x}}},-\left(\bar{A}^{T} \otimes(-\underline{\boldsymbol{b}})\right)\right]$, with $\underline{\hat{\boldsymbol{x}}}_{i}=\min \left\{-\left(\underline{A}^{T} \otimes(-\underline{\boldsymbol{b}})\right)_{i},-\left(\bar{A}^{T} \otimes(-\overline{\boldsymbol{b}})\right)_{i}\right\}$ we have $\underline{\hat{\boldsymbol{x}}} \preceq_{m}-\left(\underline{A}^{T} \otimes(-\underline{\boldsymbol{b}})\right)$ and $\overline{\hat{\boldsymbol{x}}}=-\left(\bar{A}^{T} \otimes(-\overline{\boldsymbol{b}})\right)$. Since the operation $\otimes$ for matrices is consistent with respect to relation $\preceq_{m}, \underline{A} \otimes \underline{\hat{\boldsymbol{x}}} \preceq_{m} \underline{\boldsymbol{b}}$ and $\bar{A} \otimes \overline{\hat{\boldsymbol{x}}} \preceq_{m} \overline{\boldsymbol{b}}$. It follows that $[\underline{A} \otimes \underline{\hat{\boldsymbol{x}}}, \bar{A} \otimes$ $\overline{\hat{\boldsymbol{x}}}] \preceq_{I m}[\underline{\boldsymbol{b}}, \overline{\boldsymbol{b}}]$. or $[A] \bar{\otimes}[\hat{\boldsymbol{x}}] \preceq_{I m}[\boldsymbol{b}]$. Thus $[\hat{\boldsymbol{x}}]$ is a subsolution of $[A] \bar{\otimes}[\boldsymbol{x}]=[\boldsymbol{b}]$. Let interval vector $[\dot{\boldsymbol{x}}] \in I(\mathbf{R})_{\text {max }}^{n}$ is a interval subsolution of $[A] \bar{\otimes}[\boldsymbol{x}]=[\boldsymbol{b}]$, then $[A] \bar{\otimes}[\dot{\boldsymbol{x}}] \preceq_{I m}[\boldsymbol{b}]$ or $[\underline{A}, \bar{A}] \bar{\otimes}[\underline{\underline{x}}, \overline{\boldsymbol{x}}] \preceq_{\operatorname{Im}}[\underline{\boldsymbol{b}}, \overline{\boldsymbol{b}}]$ or $[\underline{A} \otimes \underline{\boldsymbol{x}}, \bar{A} \otimes \overline{\boldsymbol{x}}] \preceq_{I m}[\underline{\boldsymbol{b}}, \overline{\boldsymbol{b}}]$, it is means that $\underline{A} \otimes \underline{\boldsymbol{x}} \preceq_{m} \underline{\boldsymbol{b}}$ and $\bar{A} \otimes \overline{\boldsymbol{x}} \preceq_{m} \overline{\overline{\boldsymbol{b}}}$. Hence, $\underline{\underline{\boldsymbol{x}}}$ and $\overline{\boldsymbol{x}}$ are a subsolution of $\underline{A} \otimes \underline{x}=\underline{\boldsymbol{b}}$ and $\bar{A} \otimes \overline{\boldsymbol{x}}=\overline{\boldsymbol{b}}$, respectively. Since is $\overline{\hat{\boldsymbol{x}}}$ the greatest subsolution of $\overline{\bar{A}} \otimes \overline{\overline{\boldsymbol{x}}}=\overline{\boldsymbol{b}}, \overline{\boldsymbol{x}} \preceq_{m} \overline{\hat{\boldsymbol{x}}}$. Then, we can show that $\underline{\dot{x}} \preceq_{m} \underline{\hat{\boldsymbol{x}}}$. Suppose that $\underline{\boldsymbol{x}} \npreceq_{m} \underline{\hat{\boldsymbol{x}}}$. Since the relation $\preceq_{m}$ in $\mathbf{R}_{\max }$ is a total relation, there is an index $i$ such that $\underline{\boldsymbol{x}}_{i} \succeq_{m} \underline{\hat{\boldsymbol{x}}}_{i}=$ $\min \left\{-\left(\underline{A}^{T} \otimes(-\underline{b})\right)_{i},-\left(\bar{A}^{T} \otimes(-\overline{\boldsymbol{b}})\right)_{i}\right\}$. It means that $(a) \underline{\boldsymbol{x}}_{i} \succ_{m}-\left(\underline{A}^{T} \otimes(-\underline{\boldsymbol{b}})\right)_{i}$ which contradicts the fact that $-\left(\underline{A}^{T} \otimes(-\underline{\boldsymbol{b}})\right)$ is the greatest subsolution system $\underline{A} \otimes \underline{\boldsymbol{x}}=\underline{\boldsymbol{b}}$, or $(b) \underline{x}_{i} \succ_{m}-\left(\bar{A}^{T} \otimes(-\overline{\boldsymbol{b}})\right)_{i}$ which contradicts that $\underline{\dot{x}} \preceq_{m} \overline{\boldsymbol{x}} \preceq_{m} \overline{\hat{\boldsymbol{x}}}=-\left(\bar{A}^{\bar{T}} \oplus(-\overline{\boldsymbol{b}})\right)$. Thus, $\underline{x} \preceq_{m} \underline{\hat{x}}$. Since $\underline{x} \preceq_{m} \underline{\hat{x}}$ and $\underline{x} \preceq_{m} \overline{\hat{x}},[\underline{\hat{x}}, \overline{\hat{x}}] \preceq_{I m}[\underline{\hat{x}}, \overline{\hat{\boldsymbol{x}}}]$. Hence, it is proved that $[\hat{\boldsymbol{x}}] \approx[\underline{\hat{x}}, \overline{\hat{\boldsymbol{x}}}]$ is the greatest subsolution of $[A] \bar{\otimes}[\boldsymbol{x}]=[\boldsymbol{b}]$.

If the greatest interval subsolution was satisfies the system, then it is an interval solution of the system. In the further discussion, we assume that the reader know about some basic concepts in fuzzy set and fuzzy number. Further details can be found in [5] and [13].

Theorem 2.4. (Decomposition Theorem) [13] If $A^{\alpha}$ is an $\alpha$-cut of fuzzy set $\tilde{A}$ in $X$ and $\tilde{A}^{\alpha}$ is a fuzzy set in $X$ with membership function $\mu(x)=\alpha \chi_{A^{\alpha}}$, where $\chi_{A^{\alpha}}$ is the charateristic function of set $A$, then $\tilde{A}=\bigcup_{\alpha \in[0,1]} \tilde{A}^{\alpha}$.

Definition 2.5. Let $\mathrm{F}(\mathbf{R})_{\tilde{\varepsilon}}:=\mathrm{F}(\mathbf{R}) \cup\{\tilde{\varepsilon}\}$, where $\mathrm{F}(\mathbf{R})$ is the set of all fuzzy numbers and $\tilde{\varepsilon}:=\{-\infty\}$, with the $\alpha$-cut of $\tilde{\varepsilon}$ is $\varepsilon^{\alpha}=[-\infty,-\infty]$ for every $\alpha \in[0,1]$. Define the operations $\tilde{\oplus}$ and $\tilde{\otimes}$ such that for every $\tilde{a}, \tilde{b} \in \mathrm{F}(\mathbf{R})_{\tilde{\varepsilon}}$ with $a^{\alpha}=\left[\underline{a}^{\alpha}, \bar{a}^{\alpha}\right] \in$ $I(\mathbf{R})_{\max }$ and $b^{\alpha}=\left[\underline{b}^{\alpha}, \bar{b}^{\alpha}\right] \in I(\mathbf{R})_{\max }:$ (i) Maximum of $\tilde{a}$ and $\tilde{b}$, written $\tilde{a} \tilde{\oplus} \tilde{b}$, is a fuzzy number whose $\alpha$-cut is interval $\left[\underline{a}^{\alpha} \oplus \underline{b}^{\alpha}, \bar{a}^{\alpha} \oplus \bar{b}^{\alpha}\right]$ for every $\alpha \in[0,1]$ (ii) Addition of $\tilde{a}$ and $\tilde{b}$, written $\tilde{a} \tilde{\otimes} \tilde{b}$, is a fuzzy number whose $\alpha$-cut is the interval $\left[\underline{a}^{\alpha} \otimes \underline{b}^{\alpha}, \bar{a}^{\alpha} \oplus \bar{b}^{\alpha}\right]$ for every $\alpha \in[0,1]$.

We can show that $\alpha$-cuts in this definition satisfy the conditions of $\alpha$-cuts of a fuzzy number. Since $\left(\left(I(\mathbf{R})_{\varepsilon}, \bar{\oplus}, \bar{\otimes}\right)\right.$ is an idempotent comutative semiring, from the operations in $F(\mathbf{R})_{\tilde{\varepsilon}}$, we can show that $\left(F(\mathbf{R})_{\tilde{\varepsilon}}, \tilde{\oplus}, \tilde{\otimes}\right)$ is an idempotent comutative semiring, with neutral element $\tilde{\varepsilon}$ and unity element $\tilde{e}=\{0\}$, with $e^{\alpha}=[0,0]$ for 
every $\alpha \in[0,1]$. The idempotent comutative semiring $F(\mathbf{R})_{\max }:=\left(F(\mathbf{R})_{\tilde{\varepsilon}}, \tilde{\oplus}, \tilde{\otimes}\right)$ is called the fuzzy number max-plus algebra and is written $F(\mathbf{R})_{\max }$.

Definition 2.6. Define $F(\mathbf{R})_{\max }^{m \times n}:=\left\{\tilde{A}=\left(\tilde{A}_{i j}\right) \mid \tilde{A}_{i j} \in F(\boldsymbol{R})_{\max }\right.$, for $i=1,2, \ldots, m$ and $j=1,2, \ldots, n\}$. The elements of $F(\mathbf{R})_{\max }^{m \times n}$ are called matrices over fuzzy number max-plus algebra.

Such matrices will be called fuzzy number matrix. The operations $\tilde{\oplus}$ and $\tilde{\otimes}$ in $F(\mathbf{R})_{\max }$ can be extended to the operations of fuzzy number matrices in $F(\mathbf{R})_{\max }^{n \times n}$. Specifically, for the matrices $\tilde{A}, \tilde{B} \in F(\mathbf{R})_{\max }^{n \times n}$, we define

$$
(\tilde{A} \tilde{\oplus} \tilde{B})_{i j}=\tilde{A}_{i j} \tilde{\oplus} \tilde{B}_{i j},(\tilde{A} \tilde{\otimes} \tilde{B})_{i j}=\bigoplus_{k=1}^{n} \tilde{A}_{i k} \tilde{\otimes} \tilde{B}_{k j} .
$$

For every $\tilde{A} \in F(\mathbf{R})_{\max }^{n \times n}$ and number $\alpha \in[0,1]$ define $\alpha$-cut matrix of matrix $\tilde{A}$, that is the interval matrix $A^{\alpha}:=\left(A_{i j}^{\alpha}\right)$, with $A_{i j}^{\alpha}$ is the $\alpha$-cut of $\tilde{A}_{i j}$ for every $i$ and $j$. Note that matrix $\underline{A^{\alpha}}=\left(\underline{A_{i j}^{\alpha}}\right) \in \mathbf{R}_{\max }^{m \times n}$ and $\overline{A^{\alpha}}=\left(\overline{A_{i j}^{\alpha}}\right) \in \mathbf{R}_{\max }^{m \times n}$ are lower bound and upper bound of matrix $\overline{A^{\alpha}}$, respectively. We can conclude that the matrices $\tilde{A}, \tilde{B} \in F(\mathbf{R})_{\max }^{m \times n}$, are equal if and only if $A^{\alpha}=B^{\alpha}$, that is $A_{i j}^{\alpha}=B_{i j}^{\alpha}$ for every $\alpha \in[0,1]$ and for every $i$ and $j$. For every fuzzy number matrix $\tilde{A}, A^{\alpha} \approx\left[\underline{A^{\alpha}}, \overline{A^{\alpha}}\right]$. Specifically, for the matrices $\tilde{A}, \tilde{B} \in F(\mathbf{R})_{\max }^{n \times n}$, we can show that $(A \oplus B)^{\alpha} \approx$ $\left[\underline{A^{\alpha}} \oplus \underline{B^{\alpha}}, \overline{A^{\alpha}} \oplus \overline{B^{\alpha}}\right]$ and $(A \otimes B)^{\alpha} \approx\left[\underline{A^{\alpha}} \otimes \underline{B^{\alpha}}, \overline{A^{\alpha}} \otimes \overline{B^{\alpha}}\right]$ for every $\alpha \in[0,1]$.

Define $F(\mathbf{R})_{\max }^{n}:=\left\{\tilde{\boldsymbol{x}}=\left[\tilde{x}_{1}, \tilde{x}_{1}, \ldots, \tilde{x}_{n}\right]^{T} \mid \tilde{x}_{i} \in F(\mathbf{R})_{\max }, i=1,2, \ldots, n\right\}$. The elements in $F(\mathbf{R})_{\max }^{n}$ are called fuzzy number vectors over $F(\mathbf{R})_{\max }$, or shortly fuzzy number vectors.

Definition 2.7. Given $\tilde{A} \in F(\mathbf{R})_{\max }^{m \times n}$ and $\tilde{\boldsymbol{b}} \in F(\mathbf{R})_{\max }^{m}$. A fuzzy number vector $\tilde{\boldsymbol{x}}^{*} \in F(\mathbf{R})_{\max }^{n}$ is called fuzzy number solution of system $\tilde{A} \tilde{\otimes} \tilde{\boldsymbol{x}}=\tilde{\boldsymbol{b}}$ if $\tilde{\boldsymbol{x}}^{*}$ satisfies the system. A fuzzy number vector $\tilde{\tilde{\boldsymbol{x}}} \in F(\mathbf{R})_{\max }^{n}$ is called fuzzy number subsolution of the system if $\tilde{A} \tilde{\otimes} \tilde{\boldsymbol{x}} \preceq_{F m} \tilde{\boldsymbol{b}}$.

Definition 2.8. Given $\tilde{A} \in F(\mathbf{R})_{\max }^{m \times n}$ and $\tilde{\mathbf{b}} \in F(\mathbf{R})_{\max }^{m}$. A fuzzy number vector $\hat{\tilde{\boldsymbol{x}}} \in F(\mathbf{R})_{\max }^{n}$ is called greatest fuzzy number subsolution of $\tilde{A} \tilde{\otimes} \tilde{\boldsymbol{x}}=\tilde{\boldsymbol{b}}$ if $\tilde{\tilde{\boldsymbol{x}}} \preceq_{F m} \hat{\tilde{\boldsymbol{x}}}$ for every fuzzy number subsolution $\tilde{\tilde{\boldsymbol{x}}}$ of the system.

Definition 2.9. Given $\tilde{A} \in F(\mathbf{R})_{\max }^{m \times n}$ with the entries of each column are not all equal to $\tilde{\varepsilon}$ and $\tilde{\boldsymbol{b}} \in F(\mathbf{R})_{\max }^{m}$. Define a fuzzy number vector $\breve{\widetilde{\boldsymbol{x}}}$ whose components are $\breve{x}_{i}$, that is a fuzzy number with the $\alpha$-cut $\breve{x}_{i}^{\alpha}=\left[\breve{x}_{i}^{\alpha}, \breve{x}_{i}^{\alpha}\right]$. The bounds of $\breve{x}_{i}^{\alpha}$ are defined recursively as bellow. Let $\underline{\hat{x}_{i}^{\alpha}}=\min \left\{\left(\left(\underline{A^{\alpha}}\right)^{T} \bar{\otimes}\left(-\underline{\boldsymbol{b}^{\alpha}}\right)\right)_{i},\left(\left(\overline{A^{\alpha}}\right)^{T} \otimes\left(-\overline{\boldsymbol{b}^{\alpha}}\right)\right)_{i}\right\}$ and $\overline{\hat{x}_{i}^{\alpha}}=-\left(\left(\overline{A^{\alpha}}\right)^{T} \otimes\left(-\overline{b^{\alpha}}\right)\right)_{i}$, 


$$
\begin{aligned}
& \underline{\breve{x}_{i}^{\alpha}}= \begin{cases}\min _{\beta \in[0,1]}\left\{\overline{\hat{x}}_{i}^{\beta}\right\}, & \text { if } \min _{\beta \in[0,1]}\left\{\overline{\hat{x}_{i}^{\beta}}\right\} \preceq_{\mathrm{m}} \underline{\hat{x}_{i}^{\alpha}} \\
\left\{\begin{array}{l}
\hat{x}_{i}^{\beta}, \text { if } \hat{x}_{i}^{\beta} \preceq_{\mathrm{m}} \hat{x}_{i}^{\alpha} \\
\overline{\hat{x}_{i}^{\alpha}}, \text { if } \hat{x}_{i}^{\beta} \succ_{\mathrm{m}} \hat{x}_{i}^{\alpha}
\end{array}, \forall \alpha, \beta \in[0,1] \text { with } \alpha>\beta,\right. & \text { if } \underline{\hat{x}_{i}^{\alpha}} \prec_{\mathrm{m}} \min _{\beta \in[0,1]}\left\{\overline{\hat{x}_{i}^{\beta}}\right\}\end{cases} \\
& \bar{x}_{i}^{\alpha}=\left\{\begin{array}{c}
\overline{\hat{x}_{i}^{\beta}}, \text { if } \overline{\hat{x}_{i}^{\beta}} \preceq_{\mathrm{m}}{\overline{\hat{x}_{i}^{\alpha}}}_{i} \\
\hat{x}_{i}^{\alpha}, \text { if } \overline{\hat{x}_{i}^{\beta}} \succ_{\mathrm{m}} \overline{\hat{x}}_{i}^{\alpha}
\end{array} \quad, \forall \alpha, \beta \in[0,1] \text { with } \alpha<\beta\right.
\end{aligned}
$$

Notice that the $\alpha$-cut family of the components of the fuzzy number vector $\breve{\widetilde{\boldsymbol{x}}}$ as in Definition 2.9 is really an $\alpha$-cut family of a fuzzy number. That is because (i) according to the Theorem $2.3, \hat{x}_{i}^{\alpha}$ is an interval, then $\breve{x}_{i}^{\alpha}$ is also an interval, (ii) $\tilde{A} \in F(\mathbf{R})_{\max }^{m \times n}$ and $\tilde{\boldsymbol{b}} \in F(\mathbf{R})_{\max }^{m}$, hence $A_{i j}^{1} \neq \emptyset$ and $\boldsymbol{b}_{i j}^{1} \neq \emptyset$. Thus $\hat{x}_{i j}^{1} \neq \emptyset$. Meanwhile from the Definition 2.9, we have $\breve{x}_{i j}^{1}=\left[\breve{x}_{i j}^{1}, \min _{\beta \in[0,1]}\left\{\overline{\hat{x}_{i}^{\beta}}\right\}\right]$, with $\underline{\breve{x}_{i j}^{1}}=$ $\left\{\begin{array}{l}\max _{\beta \in[0,1]}\left\{\underline{\left.\hat{x}_{i}^{\beta}\right\}}, \text { if } \forall \alpha \in[0,1], \underline{\hat{x}_{i}^{\alpha}} \preceq_{m} \min _{\beta \in[0,1]}\left\{\overline{\hat{x}_{i}^{\beta}}\right\}\right. \\ \min _{\beta \in[0,1]}\left\{\overline{\hat{x}_{i}^{\beta}}\right\}, \text { if } \exists \alpha \in[0,1], \underline{\hat{x}_{i}^{\alpha}} \succ_{m} \min _{\beta \in[0,1]}\left\{\overline{\hat{x}_{i}^{\beta}}\right\}\end{array}\right.$, hence $\breve{x}_{i j}^{1} \neq \emptyset,(i i i)$ from the Definition 2.9 , it is clear that $\breve{x}_{i}^{\alpha}$ is a nested $\alpha$-cut family, $(i v) \tilde{A} \in F(\mathbf{R})_{\max }^{m \times n}$ and $\tilde{\boldsymbol{b}} \in F(\mathbf{R})_{\max }^{m}$, then $A_{i j}^{0}$ and $\boldsymbol{b}_{i j}^{1}$ are bounded, respectively. Meanwhile from the Definition 2.9, we have $\breve{x}_{i j}^{0}=\left[\breve{x}_{i j}^{0}, \overline{\tilde{x}_{i j}^{0}}\right]$, with

$$
\breve{x}_{i j}^{0}=\left\{\begin{array}{c}
\min _{\beta \in[0,1]}\left\{\overline{\hat{x}_{i}^{\beta}}\right\}, \text { if } \forall \alpha \in[0,1], \underline{\hat{x}_{i}^{\alpha}} \succeq_{m} \min _{\beta \in[0,1]}\left\{\overline{\hat{x}_{i}^{\beta}}\right\} \\
\min _{\beta \in[0,1]}\left\{\underline{\left.\hat{x}_{i}^{\beta}\right\}}, \text { if } \exists \alpha \in[0,1], \underline{\hat{x}_{i}^{\alpha}} \prec_{m} \min _{\beta \in[0,1]}\left\{\overline{\hat{x}_{i}^{\beta}}\right\}\right.
\end{array},\right.
$$

hence $\hat{x}_{i}^{0}$ is also bounded.

Further, use to Decomposition Theorem in fuzzy set, we can get the components of fuzzy number vector $\breve{\widetilde{\boldsymbol{x}}}$, that is $\breve{\widetilde{x}}_{i}=\bigcup_{\alpha \in[0,1]} \tilde{c}_{i}^{\alpha}$ where $\tilde{c}_{i}^{\alpha}$ is a fuzzy set in $\mathbf{R}$ with its membership function $\mu(x)=\alpha \chi_{c_{i}^{\alpha}}$, where $\chi_{c_{i}^{\alpha}}$ is the characteristic function of $\breve{x}_{i}^{\alpha}$. With the above defined $\breve{\widetilde{\boldsymbol{x}}}$, we have that $\breve{\widetilde{\boldsymbol{x}}}$ is the greatest fuzzy number subsolution, where $\breve{x}_{i}^{\alpha} \preceq_{I m} \hat{x}_{i}^{\alpha}$ for every $\alpha \in[0,1]$ and for every $i=1,2, \ldots, n$. The theorem below gives a condition for the existence of the greatest fuzzy number subsolution the system $\tilde{A} \tilde{\otimes} \tilde{\boldsymbol{x}}=\tilde{\boldsymbol{b}}$. We show that the fuzzy number vector as defined in the Definition 2.9 is the greatest fuzzy number subsolution of the system $\tilde{A} \tilde{\otimes} \tilde{\boldsymbol{x}}=\tilde{\boldsymbol{b}}$.

Theorem 2.10. Given $\tilde{A} \in F(\mathbf{R})_{\max }^{m \times n}$ with the entries of each column are not all equal to $\tilde{\varepsilon}$ and $\tilde{\boldsymbol{b}} \in F(\mathbf{R})_{\max }^{m}$, then the fuzzy number vector $\ddot{\widetilde{\boldsymbol{x}}}$ which whose components are defined as in Definition 2.9 is the greatest fuzzy number subsolution of $\tilde{A} \tilde{\otimes} \tilde{\boldsymbol{x}}=\tilde{\boldsymbol{b}}$.

Proof. According to the Theorem 2.3, the interval vector $\hat{\boldsymbol{x}}^{\alpha}$ whose components are $\hat{x}_{i}^{\alpha}=\left[\underline{\hat{x}_{i}^{\alpha}}, \overline{\hat{x}_{i}^{\alpha}}\right]$, with $\underline{\hat{x}_{i}^{\alpha}}=\min \left[-\left(\left(\underline{A^{\alpha}}\right)^{T} \otimes\left(-\underline{\boldsymbol{b}^{\alpha}}\right)\right)_{i},-\left(\left(\overline{A^{\alpha}}\right)^{T} \otimes\left(-\overline{\boldsymbol{b}^{\alpha}}\right)\right)_{i}\right]$, and 
$\overline{\hat{x}_{i}^{\alpha}}=-\left(\left(\overline{A^{\alpha}}\right)^{T} \otimes\left(-\overline{b^{\alpha}}\right)\right)_{i}$ for every $i=1,2, \ldots, n$, is the greatest subsolution of system $A^{\alpha} \bar{\otimes}[\boldsymbol{x}]=\boldsymbol{b}^{\alpha}$ for every $\alpha \in[0,1]$. Let $\breve{\widetilde{\boldsymbol{x}}}$ be a fuzzy number vector whose components are fuzzy number $\breve{x}_{i}$, where $\breve{x}_{i}^{\alpha}=\left[\breve{x}_{i}^{\alpha}, \breve{x}_{i}^{\alpha}\right]$ whose bounds are defined as in Definition 2.9. From the definition of $\breve{x}_{i}^{\alpha}, \breve{x}_{i}^{\alpha} \preceq_{I m} \hat{x}_{i}^{\alpha}$. Thus, $\breve{\boldsymbol{x}}^{\alpha} \preceq$ Im $\hat{\boldsymbol{x}}^{\alpha}$ for every $\alpha \in[0,1]$. Since $\hat{\boldsymbol{x}}^{\alpha}$ is the greatest subsolution of $A^{\alpha} \bar{\otimes}[\boldsymbol{x}]=\boldsymbol{b}^{\alpha}$ for every $\alpha \in[0,1], A^{\alpha} \bar{\otimes} \hat{\boldsymbol{x}}^{\alpha} \preceq_{\text {Im }} \boldsymbol{b}^{\alpha}$ for every $\alpha \in[0,1]$. Since $\breve{\boldsymbol{x}}^{\alpha} \preceq_{\text {Im }} \hat{\boldsymbol{x}}^{\alpha}$ for every $\alpha \in[0,1]$ and the operation $\bar{\otimes}$ on interval matrix is consistent with respect to the order $" \preceq_{I m} ", A^{\alpha} \bar{\otimes} \breve{\boldsymbol{x}}^{\alpha} \preceq_{I m} A^{\alpha} \bar{\otimes} \hat{\boldsymbol{x}}^{\alpha} \preceq_{I m} \boldsymbol{b}^{\alpha}$ for every $\alpha \in[0,1]$. Hence, $\tilde{A} \tilde{\otimes} \breve{\tilde{\boldsymbol{x}}} \preceq_{F m} \tilde{\boldsymbol{b}}$ so $\breve{\widetilde{\boldsymbol{x}}}$ is a subsolution of the system $\tilde{A} \tilde{\otimes} \tilde{\boldsymbol{x}}=\tilde{\boldsymbol{b}}$. Let $\tilde{\tilde{\boldsymbol{x}}} \in F(\mathbf{R})_{\max }^{n}$ be a fuzzy number subsolution of $\tilde{A} \tilde{\otimes} \tilde{\boldsymbol{x}}=\tilde{\boldsymbol{b}}$, then $\tilde{A} \tilde{\otimes} \breve{\widetilde{\boldsymbol{x}}} \preceq_{F m} \tilde{\boldsymbol{b}}$ or $A^{\alpha} \bar{\otimes} \dot{\boldsymbol{x}}^{\alpha} \preceq_{I m} \boldsymbol{b}^{\alpha}$, for every $\alpha \in[0,1]$. Since $\hat{\boldsymbol{x}}^{\alpha}$ is the greatest subsolution of system $A^{\alpha} \bar{\otimes}[\boldsymbol{x}]=\boldsymbol{b}^{\alpha}, \dot{\boldsymbol{x}}^{\alpha} \preceq$ Im $\hat{\boldsymbol{x}}^{\alpha}$ for every $\alpha \in[0,1]$. Hence, we have $\tilde{\tilde{\boldsymbol{x}}} \preceq_{F m} \breve{\widetilde{\boldsymbol{x}}}$, that is $\dot{\tilde{x}}_{i} \preceq_{F m} \breve{\tilde{x}}_{i}$ or $\dot{x}_{i}^{\alpha} \preceq_{I m} \breve{x}_{i}^{\alpha}$ for every $\alpha \in[0,1]$. Suppose there exists $\alpha_{k} \in[0,1]$ such that $\dot{x}_{i}^{\alpha_{k}} \nprec_{\text {Im }} \breve{x}_{i}^{\alpha_{k}}$, that is $\dot{x}_{i}^{\alpha_{k}} \succ_{m} \breve{x}_{i}^{\alpha_{k}}$ or $\overline{\dot{x}_{i}^{\alpha_{k}}} \succ_{m} \overline{\breve{x}_{i}^{\alpha_{k}}}$. (i) For case $\dot{x}_{i}^{\alpha_{k}} \succ_{m} \breve{x}_{i}^{\alpha_{k}}$, there are three possibilities: (a) $\overline{\breve{x}_{i}^{\alpha_{k}}} \prec_{m} \underline{\hat{x}_{i}^{\alpha_{k}}} \prec_{m} \underline{x_{i}^{\alpha_{k}}}$. This leads to $\underline{\boldsymbol{x}^{\alpha_{k}}} \underline{\aleph_{m}} \underline{\hat{\boldsymbol{x}}^{\alpha_{k}}}$. Since $\underline{\hat{\boldsymbol{x}}^{\alpha_{k}}}$ is the greatest subsolution of system $\underline{A^{\alpha_{k}}} \otimes \boldsymbol{x}=\underline{\boldsymbol{b}^{\alpha_{k}}}, \underline{A^{\alpha_{k}}} \otimes \underline{\boldsymbol{x}^{\alpha_{k}}} \npreceq_{m} \underline{\boldsymbol{b}^{\alpha_{k}}}$. This contradicts the fact that $\tilde{\tilde{\boldsymbol{x}}}$ is a fuzzy number subsolution of the system $\tilde{A} \tilde{\otimes} \tilde{\boldsymbol{x}}=\tilde{\boldsymbol{b}}$. (b) Suppose that $\breve{x}_{i}^{\alpha_{k}} \prec_{m} \dot{x}_{i}^{\alpha_{k}} \prec_{m} \hat{x}_{i}^{\alpha_{k}}$. Based on the Definition 2.9, for $\breve{x}_{i}^{\alpha_{k}}$, there

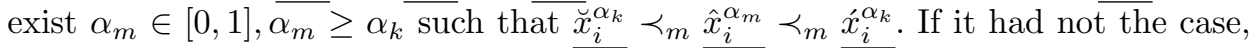
then $\underline{\breve{x}_{i}^{\alpha_{k}}} \prec_{m} \underline{\hat{x}_{i}^{\alpha_{k}}} \prec_{m} \underline{\dot{x}_{i}^{\alpha_{k}}}$, that contradicts the assumption above. Further, since the $\alpha$-cut family vector $\tilde{\tilde{\boldsymbol{x}}}$ 's component are nested, $\dot{x}_{i}^{\alpha_{k}} \preceq_{m} \dot{x}_{i}^{\alpha_{m}}$. Since $\hat{x}_{i}^{\alpha_{m}} \preceq_{m} \dot{x}_{i}^{\alpha_{k}}$ and $\underline{x}_{i}^{\alpha_{k}} \preceq_{m} \dot{x}_{i}^{\alpha_{m}}, \underline{\hat{x}^{\alpha_{m}}} \preceq_{m} \hat{x}_{i}^{\alpha_{m}}$. This leads to $\overline{\hat{\boldsymbol{x}}^{\alpha_{m}}} \npreceq_{m} \overline{\hat{\boldsymbol{x}}^{\alpha_{m}}}$. Since $\hat{\boldsymbol{x}}^{\alpha_{m}}$ is the greatest subsolution of the system $\underline{A^{\alpha_{m}}} \otimes \boldsymbol{x}=\underline{\boldsymbol{b}^{\alpha_{m}}}, \underline{A^{\alpha_{m}}} \otimes \underline{\boldsymbol{x}^{\alpha_{m}}} \npreceq_{m} \underline{\boldsymbol{b}^{\alpha_{m}}}$. It contradicts the fact that $\tilde{\tilde{\boldsymbol{x}}}$ is a fuzzy number subsolution of the system $\tilde{A} \tilde{\otimes} \tilde{\boldsymbol{x}}=\tilde{\boldsymbol{b}}$. (c). Suppose $\underline{\breve{x}_{i}^{\alpha_{k}}}=\min _{\beta \in[0,1]}\left\{\overline{\hat{x}_{i}^{\beta}}\right\} \prec_{m} \underline{x_{i}^{\alpha_{k}}} \preceq_{m} \underline{\hat{x}_{i}^{\alpha_{k}}}$. Since the $\alpha$-cuts of vector $\tilde{\tilde{x}}^{\prime} \mathrm{s}$ component are an interval, $\hat{x}_{i}^{\alpha_{k}} \preceq_{m} \overline{\hat{x}_{i}^{\alpha_{k}}}$. According to the Definition 2.9, there

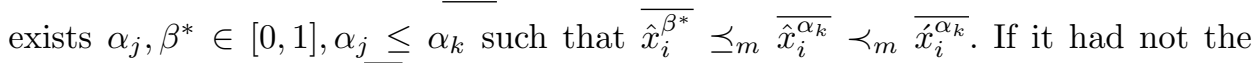
case then $\underline{\breve{x}_{i}^{\alpha_{k}}} \neq \min _{\beta \in[0,1]}\left\{\overline{\hat{x}_{i}^{\beta}}\right\}$, that contradicts the assumption above. Further, since $\alpha$-cut family of component of fuzzy number vector $\dot{\tilde{\boldsymbol{x}}}$ are nested, $\overline{\hat{x}_{i}^{\alpha_{k}}} \preceq_{m} \overline{\bar{x}_{i}^{\alpha_{j}}}$. Since $\overline{\hat{x}_{i}^{\alpha_{j}}} \prec_{m} \overline{\hat{x}_{i}^{\alpha_{k}}}$, and $\overline{x_{i}^{\alpha_{k}}} \preceq_{m} \overline{\hat{x}_{i}^{\alpha_{j}}}, \overline{x_{i}^{\alpha_{j}}} \preceq_{m} \overline{\bar{x}_{i}^{\alpha_{j}}}$, hence $\overline{\hat{\boldsymbol{x}}^{\alpha_{j}}} \npreceq_{m} \overline{\hat{\boldsymbol{x}}^{\alpha_{j}}}$. Since $\overline{\hat{\boldsymbol{x}}^{\alpha_{j}}}$ is the greatest subsolution of the system $\overline{A^{\alpha_{j}}} \otimes \boldsymbol{x}=\overline{\boldsymbol{b}^{\alpha_{j}}}, \overline{A^{\alpha_{j}}} \otimes \overline{\boldsymbol{x}^{\alpha_{j}}} \npreceq_{j} \overline{\boldsymbol{b}^{\alpha_{j}}}$. This conclusion contradicts the fact that $\tilde{\boldsymbol{x}}$ is a fuzzy number subsolution of the system $\tilde{A} \tilde{\otimes} \tilde{\boldsymbol{x}}=\tilde{\boldsymbol{b}}$. (ii) For case $\overline{\hat{x}^{\alpha_{k}}} \succ_{m} \overline{\breve{x}^{\alpha_{k}}}$ there are two possibilities: (a) Suppose $\overline{\breve{x}^{\alpha_{k}}} \prec_{m}$ $\overline{\hat{x}^{\alpha_{k}}} \prec_{m} \overline{\hat{x}^{\alpha_{k}}}$, then $\overline{\dot{\boldsymbol{x}}^{\alpha_{k}}} \npreceq_{m} \overline{\hat{\boldsymbol{x}}^{\alpha_{k}}}$. Since $\overline{\hat{\boldsymbol{x}}^{\alpha_{k}}}$ is the greatest subsolution of the system $\overline{A^{\alpha_{k}}} \otimes \boldsymbol{x}=\overline{\boldsymbol{b}^{\alpha_{k}}}, \overline{A^{\alpha_{k}}} \otimes \overline{\boldsymbol{x}^{\alpha_{k}}} \npreceq_{m} \overline{\boldsymbol{b}^{\alpha_{k}}}$ This result contradicts the fact that $\tilde{\tilde{\boldsymbol{x}}}$ is a fuzzy number subsolution of the system $\tilde{A} \tilde{\otimes} \tilde{\boldsymbol{x}}=\tilde{\boldsymbol{b}}$. (b) Suppose $\overline{\breve{x}_{i}^{\alpha_{k}}} \prec_{m} \overline{\bar{x}_{i}^{\alpha_{k}}} \prec_{m} \overline{\hat{x}_{i}^{\alpha_{k}}}$. Based on Definition 2.9 on $\overline{\breve{x}_{i}^{\alpha_{k}}}$, there exist $\alpha_{j} \in[0,1], \alpha_{j} \leq \alpha_{k}$ such that ${\overline{x_{i} \alpha_{k}}}_{\prec_{m}}$ $\overline{\hat{x}_{i}^{\alpha_{j}}} \prec_{m} \overline{\hat{x}_{i}^{\alpha_{k}}}$. If it had not the case, then $\overline{\hat{x}_{i}^{\alpha_{k}}} \prec_{m} \overline{\breve{x}_{i}^{\alpha_{k}}} \prec_{m} \overline{\hat{x}_{i}^{\alpha_{k}}}$, that contradicts the assumption above. Further, since $\alpha$-cut family of vector $\hat{\tilde{x}}^{\prime}$ 's component are nested, 
$\overline{\hat{x}_{i}^{\alpha_{k}}} \preceq_{m} \overline{\bar{x}_{i}^{\alpha_{j}}}$. Since $\overline{\hat{x}_{i}^{\alpha_{j}}} \prec_{m} \overline{\hat{x}_{i}^{\alpha_{k}}}$ and $\overline{x_{i}^{\alpha_{k}}} \prec_{m} \overline{\bar{x}_{i}^{\alpha_{j}}}, \overline{\hat{x}_{i}^{\alpha_{j}}} \prec_{m} \overline{\breve{x}_{i}^{\alpha_{j}}}$. Thus, $\overline{\hat{\boldsymbol{x}}^{\alpha_{j}}} \npreceq_{m} \overline{\hat{\boldsymbol{x}}^{\alpha_{j}}}$. Since $\overline{\hat{\boldsymbol{x}}^{\alpha_{j}}}$ is the greatest subsolution of the system $\overline{A^{\alpha_{j}}} \otimes \boldsymbol{x}=\overline{\boldsymbol{b}^{\alpha_{j}}}, \overline{A^{\alpha_{j}}} \otimes \overline{\boldsymbol{x}^{\alpha_{j}}} \npreceq_{m}$ $\overline{\boldsymbol{b}^{\alpha_{j}}}$ This result contradicts the fact that $\tilde{\tilde{\boldsymbol{x}}}$ is a fuzzy number subsolution of the system $\tilde{A} \tilde{\otimes} \tilde{\boldsymbol{x}}=\tilde{\boldsymbol{b}}$. Further, by Decomposition Theorem in fuzzy set, we can get the components of fuzzy number vector $\breve{\widetilde{x}}$ with its components are $\breve{\widetilde{x}}_{i}=\bigcup_{\alpha \in[0,1]} \tilde{c}_{i}^{\alpha}$ where $\tilde{c}_{i}^{\alpha}$ is a fuzzy set in $\mathbf{R}$ with its membership function $\mu(x)=\alpha \chi_{c_{i}^{\alpha}}$, where $\chi_{c_{i}^{\alpha}}$, where $\chi_{c_{i}^{\alpha}}$ is the characteristic function of $\breve{x}_{i}^{\alpha}$.

Before we give an example, we recall a special type of fuzzy numbers. A triangular fuzzy number $\tilde{a}$, written as $\operatorname{TFN}\left(a_{1}, a, a_{2}\right)$, is a fuzzy number with membership function

$$
\mu_{\tilde{a}}(x)=\left\{\begin{array}{cc}
\frac{x-a_{1}}{a-a_{1}}, & a_{1} \leq x \leq a \\
\frac{a_{2}-x}{a_{2}-a}, & a \leq x \leq a_{2} \\
0, & \text { others }
\end{array}\right.
$$

The support of $\tilde{a}$ is an open interval $\left(a_{1}, a_{2}\right)$ whose $\alpha$-cut is

$$
a^{\alpha}= \begin{cases}{\left[\left(a-a_{1}\right) \alpha+a_{1},-\left(a_{2}-a\right) \alpha+a_{2}\right]} & , \alpha \in(0,1] \\ {\left[a_{1}, a_{2}\right]} & , \alpha=0\end{cases}
$$

Example 2.11. In the matrix below, the $\operatorname{TFN}\left(a_{1}, a, a_{2}\right)$ will be written $\left(a_{1}, a, a_{2}\right)$. Let

$$
\tilde{A}=\left[\begin{array}{ccc}
(-3,-2,-1) & (4,5,6) & (\varepsilon, \varepsilon, \varepsilon) \\
(3,4,5) & (\varepsilon, \varepsilon, \varepsilon) & (-3,-2,0) \\
(4,5,6) & (7,8,10) & (6,7,8)
\end{array}\right] \text { and } \tilde{\boldsymbol{b}}=\left[\begin{array}{c}
(6,8,10) \\
(9,10,11) \\
(12,14,15)
\end{array}\right]
$$

We will determine the vector $\breve{\widetilde{\boldsymbol{x}}}$ that is the greatest fuzzy number subsolution of the system $\tilde{A} \tilde{\otimes} \tilde{\boldsymbol{x}}=\tilde{\boldsymbol{b}}$. Using a MATLAB computer program, we have the $\alpha$-cut bounds of $\breve{x}_{i}$ for $=0.00,0.05, \ldots, 0.95,1$, as in the Table 1. below. Then, we can determine that $\breve{x}_{1}, \breve{\widetilde{x}}_{2}$ and $\breve{\widetilde{x}}_{3}$ are the fuzzy numbers with the membership function are

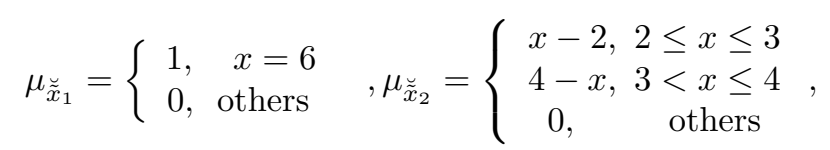

and

$$
\mu_{\breve{x}_{3}}=\left\{\begin{array}{cc}
x-6, & 6 \leq x \leq 7 \\
0, & \text { others }
\end{array},\right.
$$

respectively. We can verify that $\tilde{A} \tilde{\otimes} \breve{\boldsymbol{x}}=\tilde{\boldsymbol{b}}$, hence the greatest fuzzy number subsolution $\breve{\widetilde{\boldsymbol{x}}}$ is a fuzzy number solution of the system. 
TABLE 1 . The $\alpha$-cut bounds of $\breve{\widetilde{x}}$ 's components

\begin{tabular}{|c|c|c|c|c|c|c|}
\hline$\alpha$ & $\breve{\widetilde{x}}_{1}$ & $\overline{\widetilde{x}}_{1}$ & $\overline{\widetilde{x}}_{2}$ & $\overline{\widetilde{x}}_{2}$ & $\breve{\widetilde{x}}_{3}$ & $\overline{\widetilde{x}}_{3}$ \\
\hline 0.00 & 6.00 & 6.00 & 2.00 & 4.00 & 6.00 & 7.00 \\
0.05 & 6.00 & 6.00 & 2.05 & 3.95 & 6.05 & 7.00 \\
0.10 & 6.00 & 6.00 & 2.10 & 3.90 & 6.10 & 7.00 \\
0.15 & 6.00 & 6.00 & 2.15 & 3.85 & 6.15 & 7.00 \\
0.20 & 6.00 & 6.00 & 2.20 & 3.80 & 6.20 & 7.00 \\
0.25 & 6.00 & 6.00 & 2.25 & 3.75 & 6.25 & 7.00 \\
0.30 & 6.00 & 6.00 & 2.30 & 3.70 & 6.30 & 7.00 \\
0.35 & 6.00 & 6.00 & 2.35 & 3.65 & 6.35 & 7.00 \\
0.40 & 6.00 & 6.00 & 2.40 & 3.60 & 6.40 & 7.00 \\
0.45 & 6.00 & 6.00 & 2.45 & 3.55 & 6.45 & 7.00 \\
0.50 & 6.00 & 6.00 & 2.50 & 3.50 & 6.50 & 7.00 \\
0.55 & 6.00 & 6.00 & 2.55 & 3.45 & 6.55 & 7.00 \\
0.60 & 6.00 & 6.00 & 2.60 & 3.40 & 6.60 & 7.00 \\
0.65 & 6.00 & 6.00 & 2.65 & 3.35 & 6.65 & 7.00 \\
0.70 & 6.00 & 6.00 & 2.70 & 3.30 & 6.70 & 7.00 \\
0.75 & 6.00 & 6.00 & 2.75 & 3.25 & 6.75 & 7.00 \\
0.80 & 6.00 & 6.00 & 2.80 & 3.20 & 6.80 & 7.00 \\
0.85 & 6.00 & 6.00 & 2.85 & 3.15 & 6.85 & 7.00 \\
0.90 & 6.00 & 6.00 & 2.90 & 3.10 & 6.90 & 7.00 \\
0.95 & 6.00 & 6.00 & 2.95 & 3.05 & 6.95 & 7.00 \\
1.00 & 6.00 & 6.00 & 3.00 & 3.00 & 7.00 & 7.00 \\
\hline
\end{tabular}

\section{References}

[1] Bacelli, F., et al, Synchronization and Linearity, John Wiley and Sons, New York, 2001.

[2] Chanas, S. and Zielinski, P. 2001. "Critical path analysis in the network with fuzzy activity times", Fuzzy Sets and Systems 122 (2001), 195-204

[3] Heidergott, B., et. al, Max Plus at Work, Princeton University Press, Princeton, 2005.

[4] Krivulin, N.K., "Evaluation of Bounds on Service Cycle Times in Acyclic Fork-Join Queueing Networks", International Journal of Computing Anticipatory Systems 9 (2001), 94-109.

[5] Lee, K.H., First Course on Fuzzy Theory and Applications, Spinger-Verlag. Berlin, 2005

[6] Litvinov, G.L. and Sobolevskii, A.N. "Idempotent Interval Anaysis and Optimization Problems", Reliable Computing 7 (2001), 353-377

[7] Pardoa, M. J. and Fuente, D. de la, "Optimizing a priority-discipline queueing model using fuzzy set theory", Computers and Mathematics with Applications 54 (2007), 267-281.

[8] Ping, W.X., "Infinite fuzzy relational equations on a complete, Brouwerian lattice", Fuzzy Sets and Systems 138 (2003), 657-666.

[9] Qu, X. B. and Wang, X.P., "Some properties of infinite fuzzy relational equations on complete Brouwerian lattices", Fuzzy Sets and Systems 158 (2007), 1327-1339.

[10] Soltoni, A. and Haji, R., 2007. "A Project Scheduling Method Based on Fuzzy Theory", Journal of Industrial and Systems Engineering 1 (2007), 70-80.

[11] Wu, Y.K. and Guu, S.M., "Finding the complete set of minimal solutions for fuzzy relational equations with max-product composition", International Journal of Operations Research 1 (2004), 29-36. 
[12] Xiong, Q.Q. and Wang, X.P., "Some properties of supmin fuzzy relational equations on infinite domains", Fuzzy Sets and Systems 151 (2005), 393-402.

[13] Zimmermann, H.J. Fuzzy Set Theory and Its Applications, Kluwer Academic Publishers, Boston, 1991 